\title{
BITING FORCE AND CHEWING EFFICIENCY OF CONVENTIONAL AND CAD/CAM COMPLETE DENTURES. A CROSS-OVER STUDY
}

\author{
Nourhan Ahmed Ragheb* and Wafaa Ibrahim Ibrahim**
}

\begin{abstract}
Background: Many studies reported using CAD/CAM technology in implant-supported prostheses. However, few studies reported its use in complete dentures. The aim of this study is to compare the Maximum Biting Force (MBF) and chewing efficiency of complete dentures fabricated by conventional, rapid prototyping and milling techniques.

Materials and Methods: This is a clinical study with a cross-over design where 27 completely edentulous participants were divided in a random order into 3 equal groups as 1 . received conventional complete denture 2. received 3D printed complete dentures 3. received milled complete dentures. Assessment of MBF was performed after denture insertion at 1 week, 1 month and 3 months, using Flexiforce A $301{ }^{\circledR}$ force sensor. Mixing ability test was used to assess chewing efficiency 3 months after denture insertion. Repeated measure ANOVA test and Paired sample t-test were used with $\mathrm{P} \leq 0.05$ level of significance.
\end{abstract}

Results: Regarding the MBF, there was a statistically significant increase $(p<0.001)$ in the MBF at $1 \mathrm{w}, 1 \mathrm{~m}$ and $3 \mathrm{~m}$ follow-up periods in all three groups. There was a statistically significant difference in chewing efficiency between 5,10,20,30 and 50 strokes and between the three groups $(\mathrm{p}<0.001)$. MBF and chewing efficiency increased significantly $(\mathrm{p}<0.001)$ in Printed CAD/CAM and Milled CAD/CAM groups when compared to conventional complete dentures. Milled group showed a statistically significant improvement $(\mathrm{p}<0.001)$ in MBF and chewing efficiency when compared to Printed group.

Conclusion: The CAD/CAM fabricated dentures showed better results in terms of MBF and chewing efficiency compared to conventional complete denture.

KEY WORDS: CAD/CAM Complete denture, Rapid prototyping, Milling, Biting force, Chewing efficiency.

* Lecturer, Prosthodontist, Department of Prosthodontics, Faculty of Oral and Dental Medicine, Kafr El-Sheikh University, Kafr El-Sheikh, Egypt.

** Lecturer, Prosthodontist, Department of Prosthodontics, Faculty of Oral and Dental Medicine, Delta University for Science \& Technology, Mansoura, Egypt. 


\section{INTRODUCTION}

Edentulism is considered a handicap, affecting both oral function and psychologic condition of the patients. Complete dentures became very popular several decades ago with the presentation of acrylic polymers in dentistry. Dental implants have been widely used as a treatment option for completely edentulous patients, yet many patients due to financial problems, anatomic factors and systemic problems are contraindicated for this treatment option. ${ }^{1}$

Many researches were concerned with the association between masticatory function and the quality of life and general health in elderly people. ${ }^{2}$ Biting force is an essential factor in determination of oral function and influence the masticatory system to a great extent. ${ }^{3} \mathrm{MBF}$ has a direct influence on the choice of food and maintaining the masticatory performance ${ }^{4}$, consequently edentulous subjects may have a diet deficient in fibers and minerals. ${ }^{5}$

With teeth loss which is followed by prosthetic rehabilitation, the biting force is reduced by $20 \%$ $50 \%$ when compared to dentulous subjects and is associated by bone resorption. ${ }^{6}$ Another study reported that there is deterioration in the biting force and chewing efficiency in edentulous patients when compared to dentate participants due to muscle atrophy and tooth loss. ${ }^{7}$

Dental implants have been widely used as a treatment option for completely edentulous patients, yet many patients due to financial problems, anatomic factors and systemic problems are contraindicated for this treatment option. Conventional complete denture fabrication requires five or more clinical appointments including preliminary impression, final impression, bite registration, try-in of the waxed-up denture and delivery of the dentures. ${ }^{1,8}$

In addition, many laboratory steps are needed which results in increasing the time and inaccuracies during material manipulation, ${ }^{7}$ polymerization shrinkage of heat polymerized polymethyl methacrylate (PMMA) and deformation during finishing of the denture base. Inaccuracies affects retention, support and stability of the denture, and accordingly have an effect on patients' satisfaction and quality of life. ${ }^{9}$ Conventional complete denture fabrication can also result in allergic reaction in some patients due to the cytotoxicity of the residual monomer. ${ }^{10}$

Computer-aided design and computer-aided manufacturing (CAD/CAM) is a new treatment modality which was initially used for complete denture design and fabrication by Maeda et al. ${ }^{11} \mathrm{CAD} /$ CAM manufacturing techniques include subtractive and additive methods. Subtractive manufacturing comprise milling of fully polymerized cross-linked PMMA blank which shows higher mechanical and hygienic features and exhibits less residual monomer when compared to conventional PMMA. ${ }^{12}$

In rapid prototyping technique, a photosensitive liquid resin is used, applied layer by layer on a supporting structure and polymerized by an ultraviolet or a visible light source. ${ }^{13}$

Nevertheless, due to the waste of a large amount of resin material in the milling procedures, 3D prototyping has been used increasingly in dentistry. ${ }^{14}$

In an in-vitro study, it was mentioned that CAD/ CAM dentures showed better retention and less traumatic ulcers due to improved fit when compared with conventional complete dentures..$^{15}$ In another clinical study, it was shown that CAD/CAM complete dentures increase stability, retention and clinical performance owing to improved adaptation of the denture base and borders' seal. ${ }^{16}$ Additionally, high patient and clinician satisfaction has been recorded in $\mathrm{CAD} / \mathrm{CAM}$ complete dentures fabricated using milling and 3D prototyping techniques. ${ }^{17-19}$

Many studies reported the use of CAD/CAM technology in fabrication of implant-supported fixed and removable prosthesis. However, few clinical 
studies reported the use of CAD/CAM technology in fabrication of complete dentures. ${ }^{17,20}$

To date none of the previous studies investigated the effect of $\mathrm{CAD} / \mathrm{CAM}$ complete dentures fabricated by different techniques on the MBF and chewing efficiency. The aim of this study was to compare the MBF and chewing efficiency of complete dentures fabricated by conventional, rapid prototyping and milling techniques. The research hypothesis was that, there are no significant differences in MBF and chewing efficiency in complete dentures fabricated by the three methods.

\section{MATERIALS AND METHODS}

\section{Participant selection and treatment}

This study is a clinical study with a cross-over design. For this study, 27 completely edentulous participants were selected from the outpatient clinic of the Removable Prosthodontic Department, Faculty of dentistry, Kafr Elsheikh University, Egypt. Inclusion criteria are participants with class I Angle classification maxilla-mandibular relationship, normal alveolar bone volume, absence of temporomandibular joint disorders, absence of neuromuscular disorders and with no systemic problems that affect treatment steps. An informed consent was received from all participants after they were informed about the treatment interventions. This trial was approved by the ethical committee of the Faculty of Dentistry, Kafr Elsheikh University with approval number KD/07/21. The study protocol was registered on the Clinical Trials.gov website (ClinicalTrials.gov Identifier: NCT04793503)

To decrease the consequence of order of treatment on MBF, the participants were divided into 3 equal groups of 9, and were successively given the following prosthesis i) complete dentures fabricated by the Conventional technique ii) 3D Printed complete dentures iii) Milled complete dentures in a random order. All possible sequences were obtained using a quasi-random technique and a computer program (Microsoft Excel).

\section{Construction of the dentures}

\section{Construction of the conventional complete denture}

The denture was constructed using the conventional technique. ${ }^{21}$ After initial examination, the primary impression of the maxilla and mandible was made using stock trays and irreversible hydrocolloid impression material (Cavex Alginate, Holland BV). The final impressions were made with thermoplastic border molding green impression compound material (DPI Pinnacle Tracing Sticks, Mumbai, India) and wash with zinc oxide impression (Cavex, Holland and IRM; Dentsply). Bite registration was performed by proper adjustment of the lip support and incisal edge position of the maxillary occlusal rim; then, the level of the occlusal plane was determined using a Fox plate (Fox rule, Bio-Art A7 Plus, Italy). The vertical dimension of rest (VDR) was determined by measuring the distance between two dots placed on the most prominent areas on the nose and chin, while the patient was wearing the maxillary occlusal rim. Vertical dimension of occlusion was adjusted to be $3 \mathrm{~mm}$ less than the VDR. Centric relation was registered by guiding the mandible manually to the most retruded position. Midline, smile lines, and canine lines were marked on the occlusion rims. Mounting of the maxillary cast on the articulator (Bio-Art A7 Plus Articulator, Italy) was done using maxillary face bow, and the mandibular cast was mounted using the centric jaw relation record. The selected cross linked acrylic resin teeth (Vitapan ${ }^{\circledR}$; Vita Zahnfabrik-Germany) were arranged with bilateral balanced occlusion. After waxing up of the denture, it was checked in the patient's mouth then processed using compression molding technique. Conventional heat cured acrylic resin (Heraeus Kulzer GmbH, Germany) was used for the fabrication of the dentures. 


\section{Construction of the CAD/CAM $3 D$ printed denture}

The same steps of the conventional technique were performed till the adjustment of occlusion rims. A scan spray was applied to the occlusion blocks to be prepared for scanning with an optical 3D scanner (AutoScan-DS100, Shining 3D, Zhejang, China). The files were then translated into stereolithography (STL) files and the CDs were virtually designed in Dental CAD software (Exocad GmbH) as shown in (Figure 1). After minor modifications in the position of teeth, the virtual denture base was fabricated using 3D printing technique from PMMA-like resin (Dental Pink resin, HARZ Labs) and the teeth were fabricated from PMMA-like micro filled composite resin (Dental sand, HARZ Labs) and they were bonded in the recesses using auto-polymerizable resin (Heraeus Kulzer GmbH, Germany). After the denture was fabricated, it was finished and polished according to the conventional methods. (Figure 2)

Once the complete denture has been virtually designed in CAD software, a preview was sent for evaluation using 3D viewing software. After approval, the virtual dentures were fabricated by the milling machine (ExoCad 5x milling machine) from pre-polymerized acrylic resin pucks and the teeth were milled from acetal teeth discs (TSM Acetal Dental) and bonded to pins in the denture base using auto-polymerizable resin (Heraeus Kulzer GmbH, Germany).

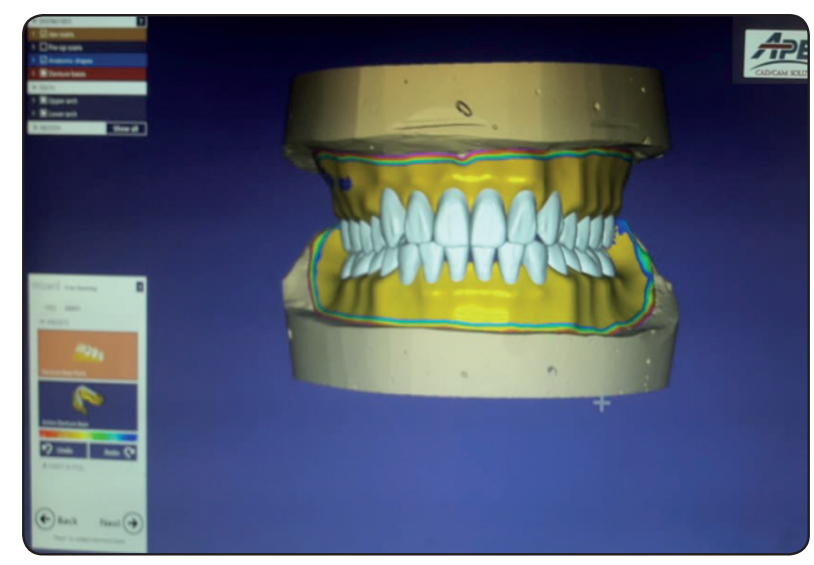

Fig. (1) Complete dentures virtually designed in Exocad Dental CAD software

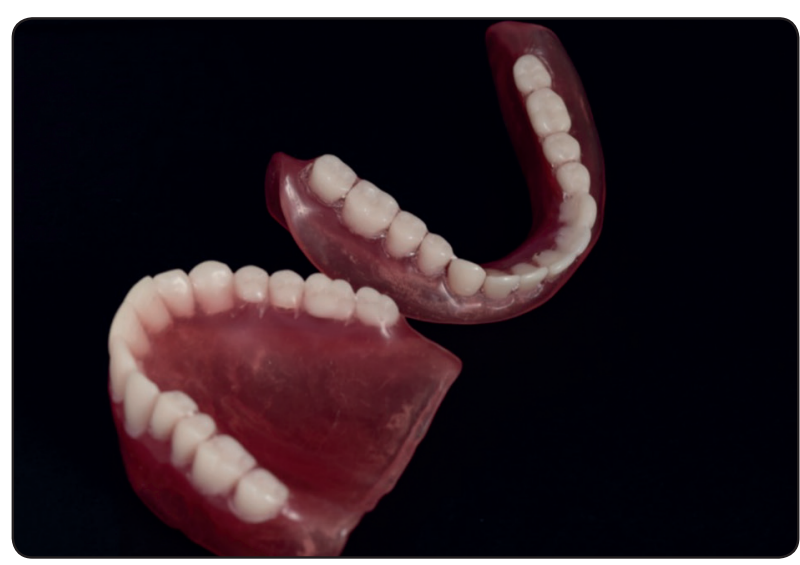

Fig. (2) CAD/CAM 3D printed dentures

For all groups, clinical assessment of the retention, support, stability, occlusion, esthetics, and phonetics was made and any necessary adjustments were carried out before delivering the dentures. Evaluation of the occlusal relationship was performed using articulating paper, and premature contacts were adjusted. A proper program for denture insertion and oral hygiene measures was given to the patients.

\section{Assessment}

\section{Assessment of biting force}

Interocclusal vertical biting force was performed bilaterally using Flexiforce A $301{ }^{\circledR}$ force sensor and Arduino microcontroller with a crystal display (Figure 3). Calibration of the sensor was performed

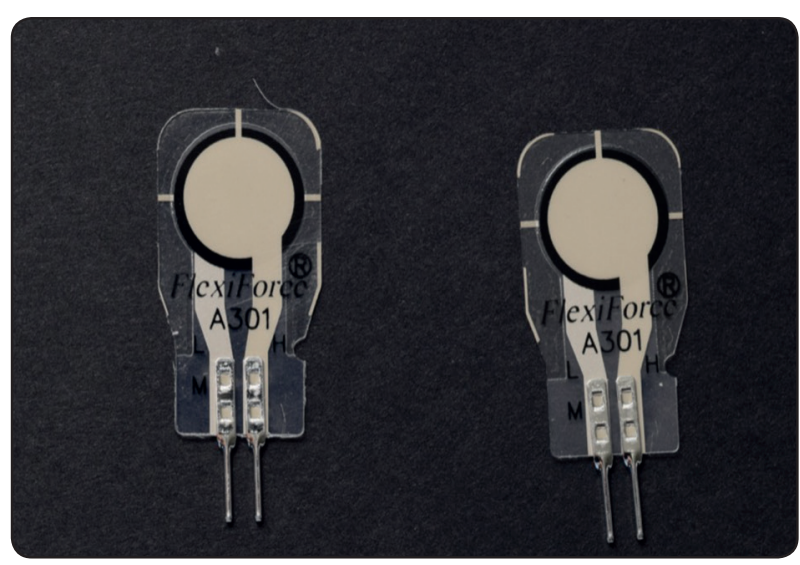

Fig. 3 Flexiforce A $301 \circledR$ force sensor 


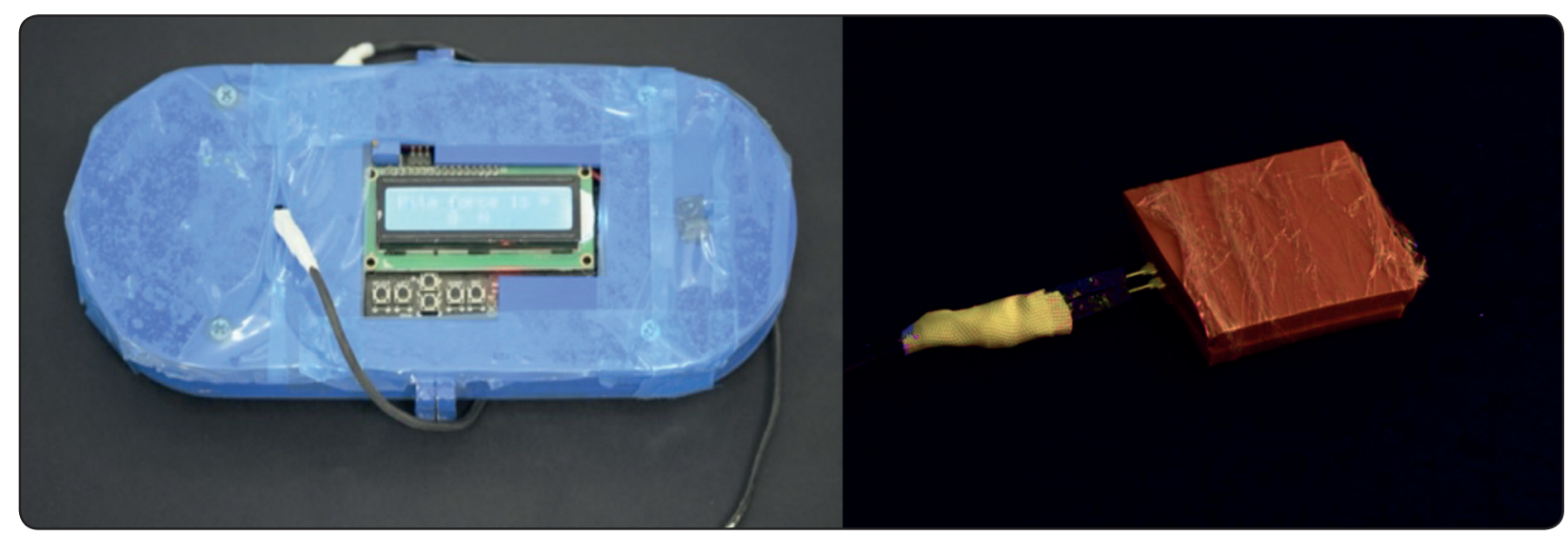

Fig. (4) A: an object with a known weight, B: 4 A layer of $3 \mathrm{~mm}$ thickness of putty rubber base impression material was used to cover the sensor

several times using an object with a known weight (Figure $4 \mathrm{~A}$ ). A layer of $3 \mathrm{~mm}$ thickness of putty rubber base impression material (Zetaplus C-silicone putty, Zhermac, Italy) was used to cover the sensor (Figure 4 B). The sensor was then placed inter-occlusally between the denture teeth at the area of upper and lower first molar. Patients were then instructed to bite as hard as possible for 3 seconds (Figure 5). The measurements were done three times with a resting period of 20 seconds between each bite. The highest biting force of the three measurements was verified, and the average bite force between the right and left sides was calculated and statistically analyzed. Assessment of biting force was performed after denture insertion at 1 week, 1 month and 3 months follow-up periods with 2 weeks wash-out period between each denture type.

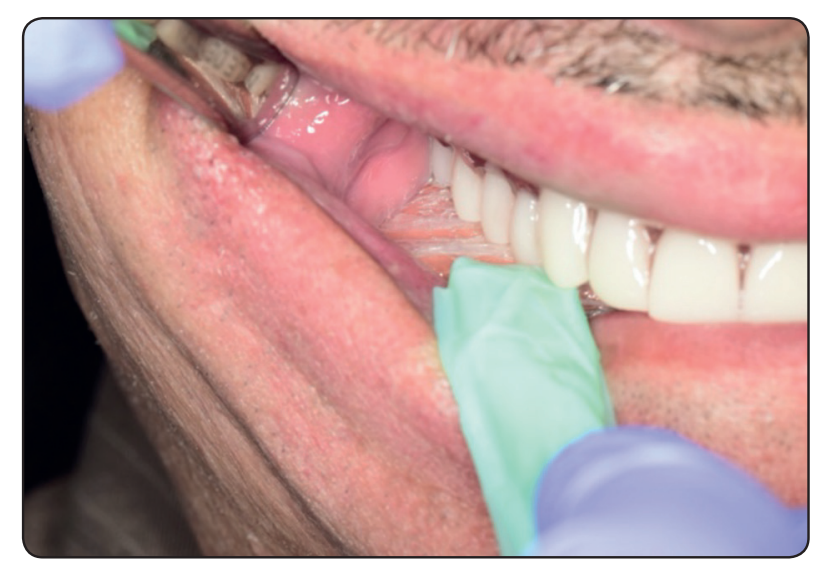

Fig. 5 Patients were then instructed to bite as hard as possible for 3 seconds

\section{Assessment of chewing efficiency}

Chewing efficiency was assessed using two-color chewing gum test. ${ }^{22}$ Two specimens of chewing gum (Trident, Mondelez Egypt Foods, Fulla gum, Egypt) with two different colors were prepared, one with a white color and the other with pink color.

Two strips of $30 \mathrm{~mm}$ are cut from each color and presented with size $30 \times 18 \times 3 \mathrm{~mm}$ and fixed together manually. Each participant was instructed to sit in an upright position in the dental chair and was asked to chew on their preferred chewing side five samples of the chewing gum for five chewing strokes of 5, 10, 20, 30 and 50 respectively. The chewing was performed at the site of the first molar. To reduce the effect of fatigue, one minute of rest was given between different cycles.

After the test, the samples were spat into transparent plastic bags. Digital scanning of the samples was performed from both sides with a digital scanner (Epson Expression 1600 Pro, Seiko Epson Corp., Nagano, Japan) with a resolution of 500 dots per inch. Scanned images were stored in Adobe Photoshop ${ }^{\circledR}$ format (Adobe Photoshop 7.0 ME®-Photo Editor Software-Adobe Systems Incorporated-USA). A reference scale was prepared by scanning a piece of unmixed chewing gum and was copied in each image (area of 4779 pixels). In the software, the magic wand tool was used 
(tolerances of 20,25,30) to select the unmixed pink areas of the images. For each side, the numbers of selected pixels were verified from the histogram, and mean of those images was calculated. Next, a ratio for the Unmixed Fraction (UF) was calculated using the following formula:

$\frac{\left(\text { Pixels }{ }_{\text {red side a }}+\text { Pixels }\right.}{\text { red side b })-2 \times \text { Pixels of scale }}$

To assess the reproducibility of the chewing process, the patients were recalled to repeat the experiment on different days. Assessment of chewing efficiency was performed 3 months after denture insertion with 2 weeks wash-out period between each denture type. All the laboratory steps were performed in the same dental laboratory. The outcome measures were assessed by two independent assessors (NA and WI).

\section{Sample size calculation}

If the response within each subject group was normally distributed with standard deviation 2.8 and if the true difference of 3.1 was considered for the estimation, we will need to study 7 in each group to be able to reject the null hypothesis that the population means are equal with probability (power) 0.8 . The Type I error probability associated with this test of this null hypothesis is 0.05 . An additional 6 patients will be added to this sample for compensation for dropouts, hence a total of 27 subjects were included.

\section{Statistical analysis}

The mean and standard deviation values were calculated for each group in each test. Data were explored for normality using Kolmogorov-Smirnov and Shapiro-Wilk tests, data showed parametric (normal) distribution.

Repeated measure ANOVA test was used to compare between more than two groups in related samples. Paired sample t-test was used to compare between two groups in related samples (Cross over study) after applying Bonferroni adjustment for multiple comparisons. The significance level was set at $\mathrm{P} \leq 0.05$. Statistical analysis was performed with IBM ${ }^{\circledR}$ SPSS $®$ Statistics Version 20 for Windows.

The whole study procedures were conducted in accordance with the CONSORT checklist.

\section{RESULTS}

The study groups consist of 27 completely edentulous participants (16 males, 11 females) with mean age $61.7 \pm 2.4$ years and the mean edentulous period of the study subjects was $5 \pm 1.3$ years. All the participants completed the follow-up period with no dropouts. Figure 6 shows the flow chart presenting different steps of the study.

\section{Biting force}

\section{Effect of time}

There was a statistically significant increase in the biting force at $1 \mathrm{w}, 1 \mathrm{~m}$ and $3 \mathrm{~m}$ follow-up periods in Conventional, Printed CAD/CAM and Milled $\mathrm{CAD} / \mathrm{CAM}$ groups, where $(\mathrm{p}<0.001)$ as shown in (Table 1, Figure 7A).

\section{Effect of groups}

For all the follow-up periods there was a statistically significant difference in biting force between Conventional, Printed CAD/CAM and Milled CADCAM groups where $(p<0.001)$ as revealed in (Table 1, Figure 7B) Biting force was significantly higher $(\mathrm{p}<0.001)$ in Printed CAD/CAM and Milled $\mathrm{CAD} / \mathrm{CAM}$ groups when compared to conventional complete dentures. Additionally, Milled group showed a statistically significant increase $(\mathrm{p}<0.001)$ in biting force when compared to Printed group.

\section{Chewing efficiency}

\section{Effect of strokes}

Regarding the Conventional, Printed CAD/ $\mathrm{CAM}$ and Milled CAD/CAM groups, there was 
a statistically significant difference between 5,10 , 20,30 and 50 strokes where $(\mathrm{p}<0.001)$ as presented in (Table 2, Figure 8A).

\section{Effect of groups}

For 5, 10, 20, 30 and 50 strokes, there was a statistically significant difference between Conventional, Printed CAD/CAM and Milled CAD/
CAM groups where $(\mathrm{p}<0.001)$ as reported in (Table 2, Figure 8B). A statistically significant difference was found between Conventional and each of Printed CAD/CAM and Milled CAD/CAM groups where $(\mathrm{p}<0.001)$. Also, a statistically significant increase in chewing efficiency was found in Milled CAD/CAM dentures when compared to Printed CAD/CAM dentures $(\mathrm{p}<0.001)$.

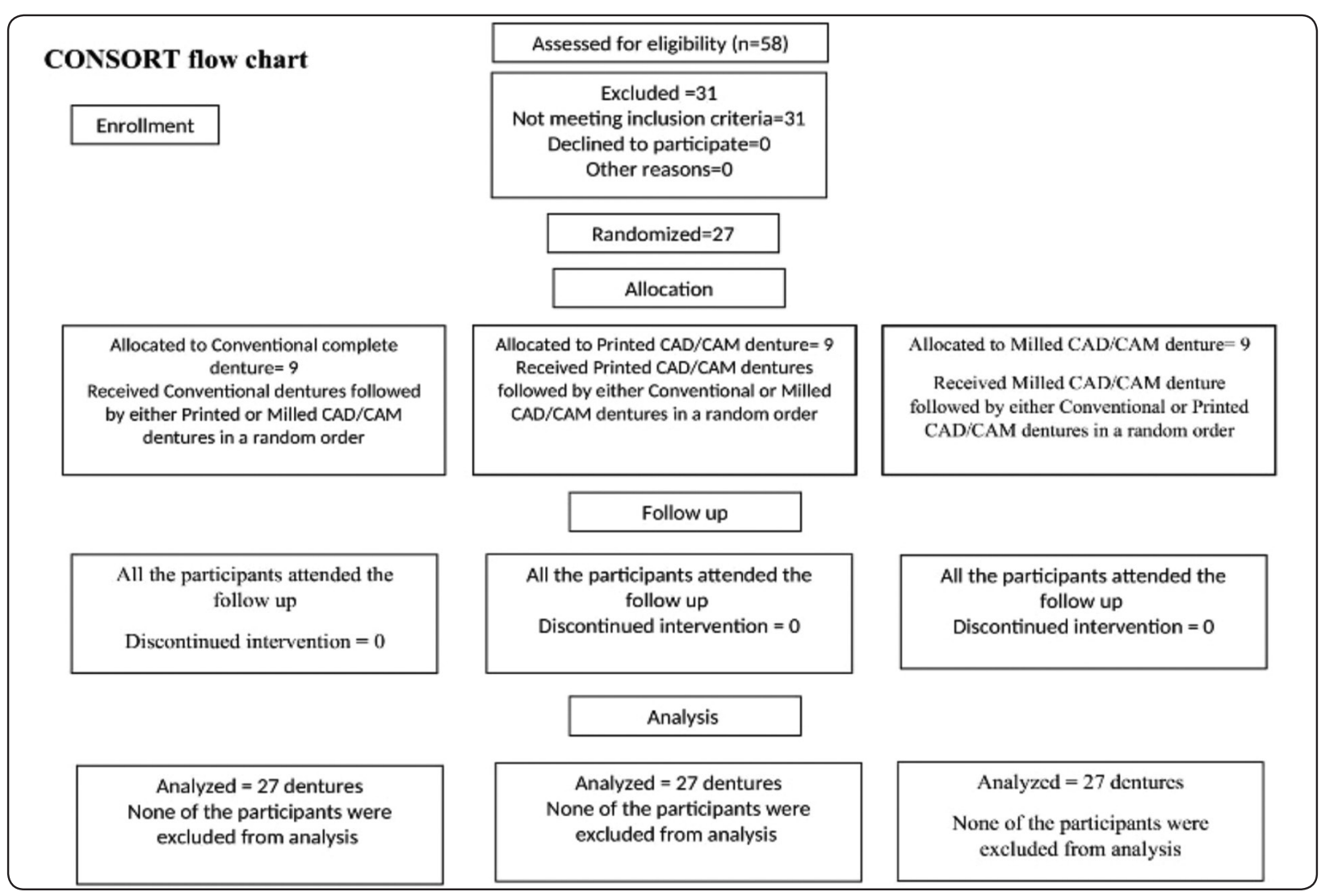

Fig. (6) Consort flow chart representing different steps of the study

TABLE (1): The mean, standard deviation (SD) values of biting force (measured in Newtons) of different groups.

\begin{tabular}{|c|c|c|c|c|c|c|c|}
\hline \multirow{3}{*}{ Variables } & \multicolumn{6}{|c|}{ Biting force } & \multirow{3}{*}{ p-value } \\
\hline & \multicolumn{2}{|c|}{ Conventional cd } & \multicolumn{2}{|c|}{ Printed cad cam } & \multicolumn{2}{|c|}{ Milled cad cam } & \\
\hline & Mean & SD & Mean & SD & Mean & SD & \\
\hline $1 \mathrm{w}$ & $288.59^{\mathrm{cC}}$ & 12.92 & $617.50^{\mathrm{cB}}$ & 11.32 & $626.59^{\mathrm{cA}}$ & 10.63 & $<0.001^{*}$ \\
\hline $1 \mathrm{~m}$ & $310.37^{\mathrm{bC}}$ & 22.28 & $634.37^{\mathrm{bB}}$ & 8.85 & $658.30^{\mathrm{bA}}$ & 10.19 & $<0.001^{*}$ \\
\hline $3 \mathrm{~m}$ & $339.15^{\mathrm{aC}}$ & 39.64 & $684.37^{\mathrm{aB}}$ & 8.36 & $717.78^{\mathrm{aA}}$ & 59.90 & $<0.001^{*}$ \\
\hline p-value & \multicolumn{2}{|c|}{$<0.001 *$} & \multicolumn{2}{|c|}{$<0.001 *$} & \multicolumn{2}{|c|}{$<0.001 *$} & \\
\hline
\end{tabular}

Means with different small letters in the same column indicates significant difference, means with different capital letters in the same row indicates significant difference *; significant $(p<0.05)$. 
TABLE (2): The mean, standard deviation (SD) values of chewing efficiency (Unmixed Fractions measured in pixels) of different groups.

\begin{tabular}{|c|c|c|c|c|c|c|c|}
\hline \multirow{3}{*}{ Variables } & \multicolumn{6}{|c|}{ Chewing efficiency } & \multirow{3}{*}{ p-value } \\
\hline & \multicolumn{2}{|c|}{ Conventional cd } & \multicolumn{2}{|c|}{ Printed cad cam } & \multicolumn{2}{|c|}{ Milled cad cam } & \\
\hline & Mean & SD & Mean & SD & Mean & SD & \\
\hline 5 strokes & $16.22^{\mathrm{aA}}$ & 0.93 & $11.81^{\mathrm{aв}}$ & 0.74 & $8.81^{\mathrm{aC}}$ & 0.68 & $<0.001 *$ \\
\hline 10 strokes & $14.22^{\mathrm{bA}}$ & 0.93 & $9.81^{\text {вв }}$ & 0.74 & $6.81^{\mathrm{bC}}$ & 0.68 & $<0.001 *$ \\
\hline 20 strokes & $12.22^{\mathrm{cA}}$ & 0.93 & $7.81^{\mathrm{cB}}$ & 0.74 & $4.89^{\mathrm{cC}}$ & 0.70 & $<0.001 *$ \\
\hline 30 strokes & $10.22^{\mathrm{dA}}$ & 0.93 & $5.81 \mathrm{~dB}$ & 0.74 & $2.89^{\mathrm{dC}}$ & 0.70 & $<0.001 *$ \\
\hline 50 strokes & $8.22^{\mathrm{eA}}$ & 0.93 & $3.81^{\mathrm{eB}}$ & 0.74 & $0.89^{\mathrm{eC}}$ & 0.70 & $<0.001 *$ \\
\hline p-value & \multicolumn{2}{|c|}{$<0.001 *$} & \multicolumn{2}{|c|}{$<0.001 *$} & \multicolumn{2}{|c|}{$<0.001 *$} & \\
\hline
\end{tabular}

Means with different small letters in the same column indicates significant difference, means with different capital letters in the same row indicates significant difference *; significant $(p<0.05)$

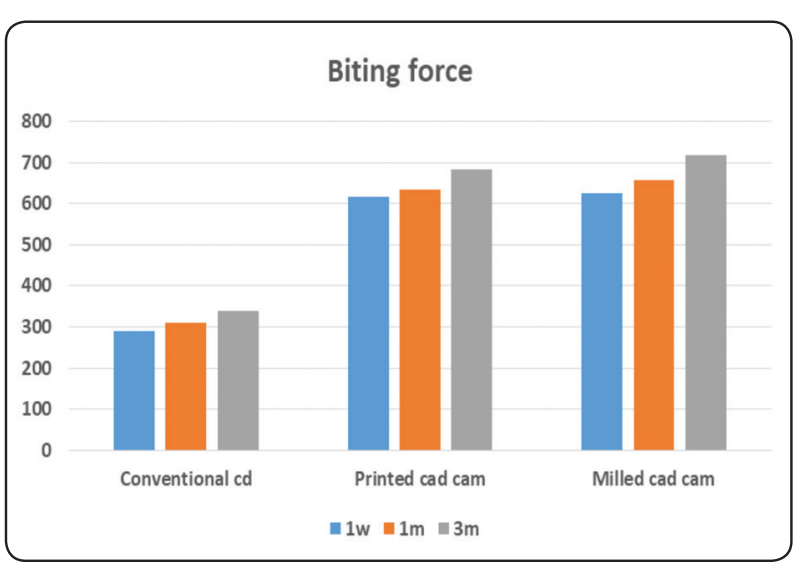

Fig. (7) Bar chart representing the biting force (in Newtons) for all three groups at all follow-up periods.

\section{DISCUSSION}

In the present study, there was a significant improvement in the biting force and chewing efficiency in CAD/CAM complete dentures. Moreover, CAD/CAM milled dentures were statistically better than rapid prototyping. Thus, the null hypothesis was rejected.

For over decades, conventional complete dentures were the only treatment option for the rehabilitation of completely edentulous patients. Yet,

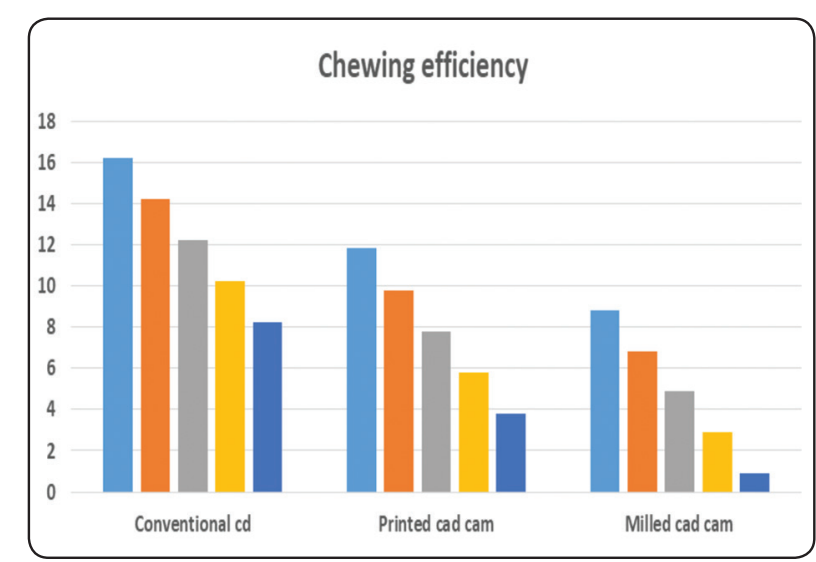

Fig. (8) Bar chart representing chewing efficiency of Unmixed Fractions measured in pixels for all three groups at all follow-up periods.

conventional complete dentures cannot completely solve psychological or functional problems. ${ }^{23}$

A crossover design was selected in the present study and the dentures were fabricated in the same dental laboratory, which helps in minimizing the variability between patients and dentures and gives more reliability to the MBF and chewing efficiency measurements. ${ }^{24}$ Previous studies showed no difference between males and females in MBF, therefore both genders were included in the present study. ${ }^{25,26}$ 
It was documented that $\mathrm{CAD} / \mathrm{CAM}$ fabricated complete dentures either by milling or rapid prototyping are to be clinically satisfactory and better than conventional methods. ${ }^{27,28}$ Although there were no previous studies comparing the MBF and chewing efficiency of conventional, milled $\mathrm{CAD} / \mathrm{CAM}$ and rapid prototyping techniques.

Previous studies ${ }^{17,29}$ compared CAD/CAM fabricated complete dentures (AvaDent digital fabrication method) with those of the conventional technique, they reported that $\mathrm{CAD} / \mathrm{CAM}$ dentures significantly have higher retention. Additionally, better patient satisfaction regarding retention, comfort, chewing efficiency, and efficiency of the technique. ${ }^{17}$ The authors explained the improved retention of digital complete dentures due to the improved fit as a result of absence of polymerization shrinkage as the dentures were milled from high density pre-polymerized blocks of acrylic resin and is not essentially influenced by hydration. ${ }^{17,29}$

One of the most important variables in masticatory performance evaluation is the MBF. ${ }^{30,31}$ Biting force differs according to its position intraorally, with the maximum force at the first molar area which is about $80 \%$ of the MBF. ${ }^{32,33}$ Therefore, several recordings were carried out in this area so that measurement are more reliable. ${ }^{34}$

Mixing ability test was used in the present study which is documented as an alternative to sieving methods. ${ }^{35}$ Sieving methods are considered by many researchers as gold standard for evaluating chewing efficiency where sieved food particles are digitally scanned by digital software. ${ }^{36}$ In this study, to test the color mixing ability two different colors of chewing gums were used to assess the chewing efficiency and the extent of color change showed the degree of mixing ability. ${ }^{22,37,38}$ Mixing ability increases when the number of chewing strokes increase and the mean ratio of unmixed fraction (UF) decrease. ${ }^{24}$ This decrease in the mean ratio is due to high degree of color mixing and a decrease in the volume of the specimens due to extraction of the sweetener. ${ }^{39}$ Both are used for measuring chewing efficiency. ${ }^{39,40}$

Chewing gums were used to evaluate chewing efficiency due to several factors. Comminution of food particles is avoided, which can stick under the denture or can be swallowed and consequently analysis will be lost. ${ }^{41}$ Besides, the chewing gum has elastic property allowing the use of the highest muscle activity. Moreover, the gums are inexpensive and easily available its chewing properties are familiar to most people. ${ }^{37,42}$

According to the results of the present study, it was observed that there was an improvement in the MBF throughout the study period regardless of the denture type. This is in agreement with a previous study $^{4}$ which showed an improvement in MBF after 1 month of denture use which was explained by the adaptation of patients to the new denture..$^{43,44}$

Additionally, the significant improvement of MBF and masticatory efficiency for the digitally fabricated dentures was attributed as mentioned by a previous study to better adaptation of CAD/CAM denture bases when compared to conventionally fabricated dentures. ${ }^{27}$ Better adaptation surely results in better retention along with better function. ${ }^{29}$ This can be explained by the fact that the cusps cut more efficiently through the food, as the artificial teeth are on a more retained and stable base..$^{45,46}$ Consequently, patients are more comfortable while chewing and exert higher biting forces. ${ }^{47}$

Moreover, the results of CAD/CAM milled complete dentures were significantly better than rapidly prototyped complete dentures in terms of MBF and chewing efficiency which can be attributed to better adaptation and significantly better trueness of the intaglio surfaces of milled CAD/CAM complete denture when compared to rapidly prototyped complete dentures, as shown by a previous invitro study. ${ }^{13}$. This could be explained by the results of previous studies as they reported that the level of maximum bite force is affected by the stability and retention of the dentures. ${ }^{48-51}$ 
In addition, $\mathrm{MBF}$ and chewing efficiency showed better results in milled dentures when compared to conventional dentures. A case study showed better retention of CAD/CAM maxillary milled complete dentures when compared to conventionally constructed dentures. The authors justified the significant improvement in denture stability, retention, besides clinical performance to good adaptation of the base and border seal of CAD/CAM complete denture ${ }^{16}$ and hence results in improvement in MBF and chewing efficiency . ${ }^{13,48-51}$

On the other hand, low MBF and masticatory efficiency of conventionally fabricated complete dentures can be explained by the dimensional changes of the denture base which usually occurs due to curing shrinkage during processing, release of internal stresses and expansion occurring as a result of water sorption. ${ }^{52}$ These changes can lead to compromised retention and stability resulting in sore spots in the mucosa. ${ }^{53}$

Although, studies that assessed the biting force and chewing efficiency of milling and 3D printing dentures are deficient, there are some new studies that can be comparable to the results of the present study. For example, Chaturvedi et al 2021a ${ }^{54}$ who assessed the occlusal force analysis (percentage of occlusal force applied on the right and left sides of the arch, centralization of forces and percentage of maximum occlusal force) in complete dentures fabricated by additive, subtractive and conventional techniques with different occlusal schemes, using computerized occlusal force analysis system [TechScan III (T-scan III), they concluded that the digital CDs fabricated by subtractive technique were demonstrated to be superior to additive technique in relations to occlusal force analysis on tested parameters. Though, further research is required on patients to determine the particular superiority of one technique over the other.

In addition, the results of the present study can be comparable to the results of Chaturvedi et al 2021b ${ }^{55}$ who compared occlusal force analysis (percentage of occlusal force applied on the right and left sides of the arch difference between them, centralization of forces and percentage of maximum occlusal/bite force ) in complete dentures (CDs) fabricated by milling, 3-D printing and conventional techniques having 3 commonly used occlusal schemes, using computerized occlusal force analysis system (Tech-Scan III- T-Scan III), they concluded that the occlusal parameters in CDs were affected by the manufacturing techniques and occlusal schemes of CDs.

The limitations of the present study include small sample size and short follow-up period. Further clinical studies with long-term follow-up period and larger number of participants are recommended for evaluation of MBF and chewing efficiency in completely edentulous patients. Additional studies are needed to evaluate if any other factors may affect the biting force and chewing efficiency like the teeth material and bonding strength between teeth and different denture base materials. Also, patient related outcomes and prosthetic complications can be assessed in further studies.

\section{CONCLUSION}

Within the limitations of the present study, when compared to conventionally fabricated complete dentures, rapidly prototyped and milled CAD/CAM complete dentures showed better results in terms of $\mathrm{MBF}$ and chewing efficiency. Milled complete dentures showed significant better results than rapidly prototyped dentures.

\section{Clinical implication}

$\mathrm{CAD} / \mathrm{CAM}$ dentures are an effective treatment option for rehabilitation of completely edentulous patients especially, if the main concern of patients is the ability of biting and chewing. 


\section{REFERENCES}

1. Jacob RF. The traditional therapeutic paradigm: complete denture therapy. J Prosthet Dent. 1998;79:6-13.

2. Kimura Y, Ogawa H, Yoshihara A, Yamaga T, Takiguchi T, Wada T, Sakamoto R, Ishimoto Y, Fukutomi E, Chen W, Fujisawa M, Okumiya K, Otsuka K, Miyazaki H, Matsubayashi K. Evaluation of chewing ability and its relationship with activities of daily living, depression, cognitive status and food intake in the communitydwelling elderly. Geriatr Gerontol Int. 2013;13:718-25.

3. Rosa LB, Bataglion C, Siéssere S, Palinkas M, Mestriner Jr W, de Freitas O, de Rossi M, de Oliveira LF, Regalo SC. Bite force and masticatory efficiency in individuals with different oral rehabilitations. Open J Stomatol. 2012; 2:21-6.

4. Borie E, Orsi IA, Fuentes R, Beltrán V, Navarro P, Pareja F, Raimundo LB. Maximum bite force in elderly indigenous and non-indigenous denture wearers. Acta Odontol Latinoam. 2014;27:115-9..

5. Allen F, McMillan A. Food selection and perceptions of chewing ability following provision of implant and conventional prostheses in complete denture wearers. Clin Oral Implants Res. 2002;13:320-6.

6. Fontijn-Tekamp FA, Slagter AP, van't Hof MA, Geertman ME, Kalk W. Bite forces with mandibular implant-retained overdentures. J Dent Res. 1998;77:1832-9.

7. Michael CG, Javid NS, Colaizzi FA, Gibbs CH. Biting strength and chewing forces in complete denture wearers. J Prosthet Dent 1990; 63:549-553.

8. Bidra AS, Taylor TD, Agar JR. Computer-aided technology for fabricating complete dentures: Historical background, current status and future perspectives. J Prosthet Dent 2013;109:361-6.

9. Darvell BW, Clark RK. The physical mechanisms of complete denture retention. Br Dent J. 2000;189:248-52.

10. Bilgin MS, Erdem A, Aglarci OS, Dilber E. Fabricating Complete Dentures with CAD/CAM and RP Technologies. J Prosthodont. 2015;24:576-9.

11. Maeda Y, Minoura M, Tsutsumi S, Okada M, Nokubi T. A CAD/CAM system for removable denture. Part I: Fabrication of complete dentures. Int J Prosthodont. 1994;7:17-21.

12. Srinivasan M, Gjengedal H, Cattani-Lorente M, Moussa M, Durual S, Schimmel M, Müller F. CAD/CAM milled complete removable dental prostheses: An in vitro evaluation of biocompatibility, mechanical properties, and surface roughness. Dent Mater J. 2018;37:526-33.

13. Kalberer N, Mehl A, Schimmel M, Müller F, Srinivasan M. CAD-CAM milled versus rapidly prototyped (3D-printed) complete dentures: An in vitro evaluation of trueness. J Prosthet Dent. 2019;121:637-43.

14. Cristache CM, Totu EE, Iorgulescu G, Pantazi A, Dorobantu D, Nechifor AC, Isildak I, Burlibasa M, Nechifor G, Enachescu M. Eighteen Months Follow-Up with Patient-Centered Outcomes Assessment of Complete Dentures Manufactured Using a Hybrid Nanocomposite and Additive CAD/CAM Protocol. J Clin Med. 2020;9:324.

15. Steinmassl O, Dumfahrt H, Grunert I, Steinmassl PA. $\mathrm{CAD} / \mathrm{CAM}$ produces dentures with improved fit. Clin Oral Investig. 2018;22:2829-35.

16. Ali MS,Al-Harbi FA. Posterior palatal seal area established in conventional and CAD/CAM fabricated complete denture techniques: Clinical Case Study. J Den Craniofac Res. 2016;1:1-6.

17. Kattadiyil MT, Jekki R, Goodacre CJ,Baba NZ.Comparison of treatment outcomes in digital and conventional complete removable dental prosthesis fabrications in a predoctoral setting. J Prosthet Dent. 2015;114:818-25.

18. Bidra AS, Farrell K, Burnham D, Dhingra A, Taylor TD, Kuo CL. Prospective cohort pilot study of 2-visit CAD/ CAM monolithic complete dentures and implant-retained overdentures: Clinical and patient-centered outcomes. J Prosthet Dent 2016;115:578-86.

19. Pereyra NM, Marano J, Subramanian G, Quek S, Leff D. Comparison of Patient Satisfaction in the Fabrication of Conventional Dentures vs. DENTCA (CAD/CAM) Dentures: A Case Report. J N J Dent Assoc. 2015;86:26-33.

20. Infante L, Yilmaz B, McGlumphy E, Finger I. Fabricating complete dentures with CAD/CAM technology. J Prosthet Dent. 2014;111:351-5.

21. Zarb, G., Bolender, C., Eckert, S., Jacob, R., Fenton, A. \& Mericske-Stern, R. Prosthodontic Treatment for Edentulous Patients. 12th ed. St Louis; Mosby; 2003.p. 211-425.

22. Schimmel M, Christou P, Herrmann F, Müller F. A twocolour chewing gum test for masticatory efficiency: development of different assessment methods. J Oral Rehabil. 2007;34:671-8 
23. de Liz Pocztaruk R, da Fontoura Frasca LC, Rivaldo EG, Mattia PR, Vidal RA, Fernandes E, Gavião MB. Satisfaction level and masticatory capacity in edentulous patients with conventional dentures and implant-retained overdentures. Braz J Oral Sci. 2006;5:1232-8.

24. Elsyad MA, Hegazy SA, Hammouda NI, Al-Tonbary GY, Habib AA. Chewing efficiency and electromyographic activity of masseter muscle with three designs of implantsupported mandibular overdentures. A cross-over study. Clin Oral Implants Res. 2014;25:742-8.

25. Soboleva U, Lauriņa L, Slaidina A. The masticatory system--an overview. Stomatologija. 2005;7:77-80.

26. Wichelhaus A, Hüffmeier S, Sander FG. Dynamic functional force measurements on an anterior bite plane during the night. J Orofac Orthop. 2003;64:417-25.

27. Goodacre BJ, Goodacre CJ, Baba NZ, Kattadiyil MT. Comparison of denture base adaptation between CADCAM and conventional fabrication techniques. J Prosthet Dent 2016;116:249-56.

28. Chen H, Wang H, Lv P, Wang Y, Sun Y. Quantitative Evaluation of Tissue Surface Adaption of CAD-Designed and 3D Printed Wax Pattern of Maxillary Complete Denture. Biomed Res Int. 2015;2015:453968

29. AlHelal A, AlRumaih HS, Kattadiyil MT, Baba NZ, Goodacre CJ. Comparison of retention between maxillary milled and conventional denture bases: a clinical study. J Prosthet Dent 2017;117:233-38.

30. Yen CI, Mao SH, Chen CH, Chen CT, Lee MY. The correlation between surface electromyography and bite force of mastication muscles in Asian young adults. Ann Plast Surg. 2015;74 Suppl 2:S168-72.

31. Koç D, Doğan A, Bek B. Effect of gender, facial dimensions, body mass index and type of functional occlusion on bite force. J Appl Oral Sci. 2011;19:274-9.

32. Shinogaya T, Sodeyama A, Matsumoto M. Bite force and occlusal load distribution in normal complete dentitions of young adults. Eur J Prosthodont Restor Dent. 1999;7: 65-70.

33. Tortopidis D, Lyons MF, Baxendale RH, Gilmour WH. The variability of bite force measurement between sessions, in different positions within the dental arch. J Oral Rehabil. 1998;25:681-6.

34. Ikebe K, Matsuda KI, Morii K, Hazeyama T, Kagawa R, Ogawa T, Nokubi T. Relationship between bite force and salivary flow in older adults. Oral Surg Oral Med Oral Pathol Oral Radiol Endod. 2007;104:510-5.

35. Wada S, Kawate N, Mizuma M. What Type of Food Can Older Adults Masticate?: Evaluation of Mastication Performance Using Color-Changeable Chewing Gum. Dysphagia. 2017;32:636-43.

36. Eberhard L, Schneider S, Eiffler C, Kappel S, Giannakopoulos NN. Particle size distributions determined by optical scanning and by sieving in the assessment of masticatory performance of complete denture wearers. Clin Oral Investig. 2015;19:429-36.

37. Elgestad Stjernfeldt P, Sjögren P, Wårdh I, Boström AM. Systematic review of measurement properties of methods for objectively assessing masticatory performance. Clin Exp Dent Res. 2019;5:76-104

38. Silva LC, Nogueira TE, Rios LF, Schimmel M, Leles CR. Reliability of a two-colour chewing gum test to assess masticatory performance in complete denture wearers. J Oral Rehabil. 2018;45:301-7.

39. Schimmel M, Leemann B, Herrmann FR, Kiliaridis S, Schnider A, Müller F. Masticatory function and bite force in stroke patients. J Dent Res. 2011;90:230-4

40. Van der Bilt A, Burgers M, van Kampen FM, Cune MS Mandibular implant-supported overdentures and oral function. Clin Oral Implants Res. 2010;21:1209-13.

41. Liedberg B, Owall B. Oral bolus kneading and shaping measured with chewing gum. Dysphagia. 1995;10:101-6.

42. van der Bilt A, Mojet J, Tekamp FA, Abbink JH. Comparing masticatory performance and mixing ability. J Oral Rehabil. 2010;37:79-84.

43. Liedberg B, Stoltze K, Owall B. The masticatory handicap of wearing removable dentures in elderly men. Gerodontology. 2005;22:10-6.

44. Müller F, Heath MR, Ott R. Maximum bite force after the replacement of complete dentures. Gerodontology. 2001;18:58-62.

45. Assunção WG, Zardo GG, Delben JA, Barão VA. Comparing the efficacy of mandibular implant-retained overdentures and conventional dentures among elderly edentulous patients: satisfaction and quality of life. Gerodontology. 2007;24:235-8.

46. Assunção WG, Barão VA, Delben JA, Gomes EA, Tabata LF. A comparison of patient satisfaction between treatment 
with conventional complete dentures and overdentures in the elderly: a literature review. Gerodontology. 2010; 27: 154-62.

47. Fontijn-Tekamp FA, Slagter AP, Van Der Bilt A, Van 'T Hof MA, Witter DJ, Kalk W, Jansen JA. Biting and chewing in overdentures, full dentures, and natural dentitions. J Dent Res. 2000;79:1519-24.

48. Haraldson T, Carlsson GE. Bite force and oral function in patients with osseointigrated oral implants. Scand J Dent Res. 1977; 86: 200-208.

49. van Kampen FMC, van der Bilt A, Cune MS, Bosman F. The influence of various attachement types in mandibular implant-retained overdentures on maximum bite force and EMG. J Dent Res.2002; 81: 170- 173.

50. Fontijn-Tekamp FA, Slagter AP, van't Hof MA, Geertman ME, Kalk W. Bite forces with mandibular implant-retained overdentures. J Dent Res. 1998; 77: 1832- 1839.

51. Carlsson GE, Lindquist LW. Ten-year longitudinal study of masticatory function in edentulous patients treated with fixed complete dentures on osseointegrated implants. Int J Prosthodont.1994; 7: 448-453.

52. Wong DM, Cheng LY, Chow TW, Clark RK. Effect of processing method on the dimensional accuracy and water sorption of acrylic resin dentures. J Prosthet Dent. 1999;81:300-4.

53. Lim SR, Lee JS. Three dimensional deformation of drystored complete denture base at room temperature. J Adv Prosthodont. 2016;8:296-303.

54. Chaturvedi S, Addas MK, Alqahtani NM, Al Ahmari NM, Alfarsi MA. Computerized occlusal forces analysis in complete dentures fabricated by additive and subtractive techniques. Technol Health Care. 2021a Mar 5. Epub ahead of print.

55. Chaturvedi S, Addas MK, Alqahtani NM, Al Ahmari NM, Alfarsi MA. Clinical analysis of CAD-CAM milled and printed complete dentures using computerized occlusal force analyser. Technol Health Care. 2021b Mar 9. Epub ahead of print. 